IJMMS 30:11 (2002) 659-665

PII. S0161171202109203

http://ijmms.hindawi.com

(c) Hindawi Publishing Corp.

\title{
ON MATLIS DUALIZING MODULES
}

\section{EDGAR E. ENOCHS, J. A. LÓPEZ-RAMOS, and B. TORRECILLAS}

Received 30 September 2001

\begin{abstract}
We consider rings admitting a Matlis dualizing module $E$. We argue that if $R$ admits two such dualizing modules, then a module is reflexive with respect to one if and only if it is reflexive with respect to the other. Using this fact we argue that the number (whether finite or infinite) of distinct dualizing modules equals the number of distinct invertible $(R, R)$-bimodules. We show by example that this number can be greater than one.
\end{abstract}

2000 Mathematics Subject Classification: 16D20, 16D90.

1. Notation and preliminaries. Throughout this paper, $R$ is always an associative ring, ${ }_{R} M$ (resp., $M_{R}$ ) means that $M$ is a left (right) $R$-module, and ${ }_{R} M_{R}$ means that $M$ is a left-right $(R, R)$-bimodule. We denote by $E(M)$ the injective envelope of the (left or right) $R$-module $M$.

We recall from [4] the following definition.

DEFINITION 1.1. A ring $R$ has a Matlis dualizing module if there is an $(R, R)$ bimodule $E$ such that ${ }_{R} E$ and $E_{R}$ are both injective cogenerators, and such that the canonical maps $R \rightarrow \operatorname{Hom}_{R}\left({ }_{R} E,{ }_{R} E\right)$ and $R \rightarrow \operatorname{Hom}_{R}\left(E_{R}, R_{R}\right)$ are both bijections.

If such $E$ exists, it will be called a Matlis dualizing module for $R$.

We note that, each of the maps

$$
R \rightarrow \operatorname{Hom}_{R}\left({ }_{R} E,{ }_{R} E\right), \quad R \rightarrow \operatorname{Hom}_{R}\left(E_{R}, R_{R}\right)
$$

is a morphism of $(R, R)$-bimodules. If $M$ is a left (right) module, when there is such a Matlis dualizing module $E$, we denote by $M^{\vee}$ the right (left) $R$-module $\operatorname{Hom}_{R}(M, E)$. If the canonical map $M \rightarrow M^{\vee \vee}$ is an isomorphism, $M$ is $E$-reflexive. If $R$ is a ring having a Matlis dualizing module, then it is clear that $R$ and $E$ are examples of $E$-reflexive modules (both of them as left and right $R$-modules).

Note that, the duality given by such a Matlis dualizing module is what in [8] and its reference is called a Morita self-duality.

Several examples of Matlis dualizing modules are given in [4]. With the following one we give a partial answer to [8, Question 4.16].

EXAMPLE 1.2. Let $R$ be a left and right Noetherian ring having a Matlis dualizing module $E$. Then, by [5, Theorem 1], $E\left[x^{-1}\right]$ is an injective cogenerator as left and right $R[[x]]$-module. Furthermore, by using [7, Theorem 4.1],

$$
\operatorname{Hom}_{R[[x]]}\left(E\left[x^{-1}\right], E\left[x^{-1}\right]\right) \cong \operatorname{Hom}_{R}(E, E)[[x]] \cong R[[x]]
$$

which shows that $E\left[x^{-1}\right]$ is a Matlis dualizing module for $R[[x]]$. 
LEMMA 1.3. If $R$ has a Matlis dualizing module $E$ and $0 \rightarrow M^{\prime} \rightarrow M \rightarrow M^{\prime \prime} \rightarrow 0$ is an exact sequence of left or right $R$-modules, then $M$ is E-reflexive if and only if $M^{\prime}$ and $M^{\prime \prime}$ are E-reflexive.

Proof. The lemma follows easily by applying the snake lemma to the commutative diagram

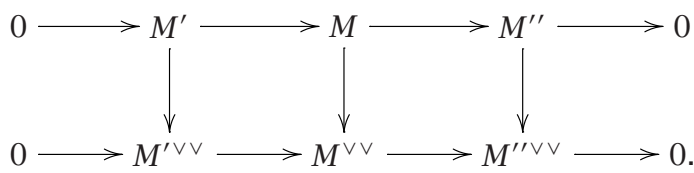

If $R$ has a Matlis dualizing module $E$, then recall from [1] that a left (right) $R$-module $M$ is finitely cogenerated by $E$ or simply finitely cogenerated if $M$ may be embedded in a finite direct sum of copies of $E$. An immediate consequence of the foregoing lemma is that every finitely generated and every finitely cogenerated left or right $R$-module is $E$-reflexive.

Osofsky in [6] showed that, if ${ }_{R} E_{S}$ defines a Morita duality, then no infinite direct sum of nonzero modules is reflexive. The following lemma is a particular case of this result. We include here a proof for completeness.

LEMMA 1.4. Let $E$ be a Matlis dualizing module for $R$ and $\left(M_{i}\right)_{i \in I}$ a family of left (right) $R$-modules. Then $\oplus_{i \in I} M_{i}$ is E-reflexive if and only if each $M_{i}$ is E-reflexive and $M_{i}=0$ except for a finite number of $i$.

Proof. If $M_{i}=0$ except for a finite number of $i$, and each $M_{i}$ is $E$-reflexive, then

$$
\operatorname{Hom}_{R}\left(\operatorname{Hom}_{R}\left(\oplus_{i \in I} M_{i}, E\right), E\right) \cong \oplus_{i \in I} \operatorname{Hom}_{R}\left(\operatorname{Hom}_{R}\left(M_{i}, E\right), E\right) \cong \oplus_{i \in I} M_{i}
$$

Conversely, we show first that no infinite direct sum of nonzero modules is $E$-reflexive. So consider $\oplus_{i \in I} M_{i}$ with $M_{i} \neq 0$ for all $i \in I$, and the canonical morphism

$$
\oplus_{i \in I} M_{i} \longrightarrow \operatorname{Hom}_{R}\left(\operatorname{Hom}_{R}\left(\oplus_{i \in I} M_{i}, E\right), E\right) \cong \operatorname{Hom}_{R}\left(\prod_{i \in I} \operatorname{Hom}_{R}\left(M_{i}, E\right), E\right),
$$

defined by

$$
\left(x_{i}\right)_{i \in I} \longrightarrow f \in \operatorname{Hom}_{R}\left(\prod_{i \in I} \operatorname{Hom}_{R}\left(M_{i}, E\right), E\right),
$$

where $f\left(\left(\Phi_{i}\right)_{i \in I}\right)=\sum_{i \in I} \Phi_{i}\left(x_{i}\right)$. The image of $\left(x_{i}\right)_{i \in I}$ is zero in $\oplus_{i \in I} \operatorname{Hom}_{R}\left(M_{i}, E\right)$ which is contained in $\prod_{i \in I} \operatorname{Hom}_{R}\left(M_{i}, E\right)$ if and only if $x_{i}=0$ for all $i \in I$, that is, $\left(x_{i}\right)_{i \in I}=$ 0 . Now since $\oplus_{i \in I} \operatorname{Hom}_{R}\left(M_{i}, E\right) \neq \prod_{i \in I} \operatorname{Hom}_{R}\left(M_{i}, E\right)$ and $E$ is a cogenerator, $\operatorname{Hom}_{R}$ $\left(\prod_{i \in I} \operatorname{Hom}_{R}\left(M_{i}, E\right) / \oplus_{i \in I} \operatorname{Hom}_{R}\left(M_{i}, E\right), E\right) \neq 0$, which means that there is a nonzero $f: \prod_{i \in I} \operatorname{Hom}_{R}\left(M_{i}, E\right) \rightarrow E$ and such that $\left.f\right|_{\oplus_{i \in I} \operatorname{Hom}_{R}\left(M_{i}, E\right)}=0$. But from the foregoing, $f$ cannot be the image of $\left(x_{i}\right)_{i \in I} \in \oplus_{i \in I} M_{i}$, and so $\oplus_{i \in I} M_{i}$ is not $E$-reflexive.

The fact that every $M_{i}$ is $E$-reflexive is a consequence of Lemma 1.3. 
The following result characterizes $E$-reflexive modules in terms of some submodule. This result is shown by using Enochs's argument in [3, Proposition 1.3] to characterize Matlis reflexive modules over a complete local Noetherian ring.

Proposition 1.5. Let $R$ be a ring having a Matlis dualizing module E. Then a left (right) $R$-module $M$ is $E$-reflexive if and only if it contains a finitely generated submodule $N$, such that $M / N$ is finitely cogenerated.

Proof. If $M$ contains such a submodule $N$, then $M$ is $E$-reflexive by using Lemma 1.3.

Conversely, if $M=0$ there is nothing to prove. If $M \neq 0$, then it contains a finitely generated submodule $N_{1}$ such that the socle of $M / N_{1}$, Soc $\left(M / N_{1}\right)$, is not zero. If $\operatorname{Soc}\left(M / N_{1}\right)$ is not essential in $M / S_{1}$, let $L / N_{1} \cap \operatorname{Soc}\left(M / N_{1}\right)=0$ with $N_{1} \subset L$, then there is a finitely generated $R$-module $N_{2}$ such that $N_{1} \subset N_{2} \subset L$, and such that $\operatorname{Soc}\left(L / N_{2}\right) \neq 0$. Then, $\operatorname{Soc}\left(M / N_{1}\right) \rightarrow \operatorname{Soc}\left(M / N_{2}\right)$ is injective but not surjective. Repeating the process we observe that we must stop, since otherwise if $T=\cup N_{n}$ then $\operatorname{Soc}(M / T)$ is an infinite direct sum, which is not possible since $M / T$ is $E$-reflexive and then $\operatorname{Soc}(M / T)$ would also be $E$-reflexive, in contradiction with Lemma 1.4. The result follows from [1, Proposition 10.7].

REMARK 1.6. Proposition 1.5 shows that the reflexive property of a module is something that depends only on the internal structure of such a module.

As a result of the preceding remark, we get the following immediate but important consequence.

COROLLARY 1.7. Let $R$ be a ring admitting several Matlis dualizing modules. Suppose that $E_{1}$ and $E_{2}$ are two of these modules and let $M$ be a left (right) $R$-module. Then $M$ is $E_{1}$-reflexive if and only if it is $E_{2}$-reflexive.

Proposition 1.8. Let $(R, M)$ be a local commutative ring having a Matlis dualizing module $E$ (not necessarily $E(R / M)$ ). Then $R$ is complete.

Proof. We show that the dual of any Artinian left (right) $R$-module $M$ is complete. If $M$ is Artinian, then it is embedded in a finite direct product of copies of $E$. Then, we have an epimorphism

$$
\operatorname{Hom}_{R}(E, E)^{n} \longrightarrow \operatorname{Hom}_{R}(M, E)
$$

for some natural number $n$, that is, $M^{\vee}$ if finitely generated. But $M^{\vee}$ has also a structure as $\hat{R}$-module since $E$ has such a structure. Then it follows immediately that $M^{\vee}$ is finitely generated as $\hat{R}$-module and so it is complete. The result follows now from the fact that there is a correspondence between submodules of $M$ and quotient modules of $M^{\vee}$ and therefore, if $M$ is Noetherian, then $M^{\vee}$ is Artinian and this is the case for $R$ and $R^{\vee} \cong E$.

REMARK 1.9. Proposition 1.8 shows that if a local commutative ring $(R, \mathcal{M})$ admits a Matlis dualizing module, then $E(R / M)$ is also Matlis dualizing (since then $R$ is complete). In this case, $E$ is a direct sum of copies of $E(R / M)$, but if the direct sum contains more than two summands, then $\operatorname{Hom}_{R}(E, E) \cong R$ would not be commutative, and so, 
$E \cong E(R / \mathcal{M})$, both as a left and as a right module, but in general $E$ is not isomorphic to $E(R / M)$ as an $(R, R)$-bimodule.

2. Matlis dualizing modules. In Section 1 we mentioned the possibility that a ring admits different and not necessarily isomorphic Matlis dualizing modules. We start this section by giving an example of a ring having more than one Matlis dualizing module.

EXAMPLE 2.1. Let $A$ be a finite-dimensional algebra over a field $k$. If we consider ${ }_{A} M$, we have the dual $M^{*}=\operatorname{Hom}_{k}(M, k)$. If $\operatorname{dim}_{k}(M)<\infty$ then $M$ is reflexive with respect to this duality (cf. [2]). From this it may be deduced that ${ }_{A} E_{A}=\operatorname{Hom}_{k}(A, k)$ is a Matlis dualizing module for $A$.

Now, let $\sigma: A \rightarrow A$ be an automorphism and let ${ }_{A} E_{A}^{\prime}$ be such that ${ }_{A} E^{\prime}={ }_{A} E$, but if $x \in E^{\prime}$ and $a \in A$, we define $x a=x \sigma(a)$ (in $E$ ). If $f:{ }_{A} E_{A} \rightarrow{ }_{A} E_{A}$ is an isomorphism (as bimodules) then $f:{ }_{A} E \rightarrow{ }_{A} E^{\prime}$ is an isomorphism, and we know that for $f(x)=x c$ for some $c \in A$. Since $f$ is an isomorphism then such a $c$ is invertible. But $f(x a)=f(x) a$ which means that $(x a) c=x c \sigma(a)$ for every $x \in E$. This gives us that $\sigma(a)=c^{-1} a c$ for all $a \in A$. Now if $\sigma$ is not of this form, we get that $E$ and $E^{\prime}$ are not isomorphic as $(A, A)$-bimodules.

In any case, $A \rightarrow \operatorname{Hom}_{A}\left({ }_{A} E^{\prime},{ }_{A} E^{\prime}\right)=\operatorname{Hom}_{A}\left({ }_{A} E,{ }_{A} E\right) \cong A$ is an isomorphism and $A \rightarrow$ $\operatorname{Hom}_{A}\left(E_{A}^{\prime}, E_{A}^{\prime}\right)$ is a monomorphism. But, since $E_{A}$ is an injective cogenerator, $E_{A}^{\prime}$ is also an injective cogenerator and if $\operatorname{Soc}\left(E_{A}^{\prime}\right)=\operatorname{Soc}\left(E_{A}\right)$, we get that $E_{A}^{\prime} \cong E_{A}$. Then

$$
\operatorname{dim}_{k}\left(\operatorname{Hom}_{A}\left(E_{A}^{\prime}, E_{A}^{\prime}\right)\right)=\operatorname{dim}_{k}\left(\operatorname{Hom}_{A}\left(E_{A}, E_{A}\right)\right)=\operatorname{dim}_{k}(A),
$$

so $A \rightarrow \operatorname{Hom}_{A}\left(E_{A}^{\prime}, E_{A}^{\prime}\right)$ is also an isomorphism and therefore ${ }_{A} E_{A}^{\prime}$ is a Matlis dualizing module for $A$.

Now, if $p$ is a prime, $k=\mathbb{Z} /(p), G$ is a commutative group such that $|G|=p^{n}$ for some positive integer $n$, and we consider group algebra $A=k G, A$ is local, so there is only one simple module over $A$. Then $\operatorname{Soc}\left(E_{A}^{\prime}\right)=\operatorname{Soc}\left(E_{A}\right)$ is simple since $A$ is commutative and we only have to find an automorphism of $A$ which is not a conjugation by any unit of $A$.

So take $p=2, k=\mathbb{Z} /(2)$ and let the group $G=H \times H$ where $H$ is the multiplicative group with two elements $H=\{1, h\}$. Take $\sigma: k G \rightarrow k G$ induced by the automorphism in $G$ given by

$$
\left(h_{1}, h_{2}\right) \longrightarrow\left(h_{2}, h_{1}\right)
$$

If $\{(1,1),(1, h),(h, 1),(h, h)\}$ is a basis for $k G$, we have that, for any element in $k G$ (in matrix notation), $\sigma((x, y, z, t))=(x, z, y, t)$, and since $k G$ is commutative, we get that $\sigma$ cannot be a conjugation for some unit of $A$.

THEOREM 2.2. Let $R$ be a ring having Matlis dualizing modules, and let $E_{1}$ and $E_{2}$ be two of such modules. Denote by $P$ and $Q$ the $(R, R)$-bimodules $\operatorname{Hom}_{R}\left(E_{1 R}, E_{2 R}\right)$ and $\operatorname{Hom}_{R}\left(E_{2 R}, E_{1 R}\right)$. Then

(i) $P \otimes_{R} Q \cong R$ and $Q \otimes_{R} P \cong R$;

(ii) $P$ and $Q$ are generators (as left and right $R$-modules); 
(iii) $P$ and $Q$ are finitely generated projective (as left and right $R$-modules);

(iv) $P \cong \operatorname{Hom}_{R}\left(Q_{R}, R_{R}\right)$ and $Q \cong \operatorname{Hom}_{R}\left(P_{R}, R_{R}\right)$.

Proof. Denote by $\operatorname{Hom}_{R}\left(E_{i}, E_{j}\right)$ the $(R, R)$-bimodule $\operatorname{Hom}_{R}\left(E_{i R}, E_{j R}\right)$.

(i) What we want to show is that the morphisms

$$
\begin{aligned}
& \operatorname{Hom}_{R}\left(E_{1}, E_{2}\right) \otimes_{R} \operatorname{Hom}_{R}\left(E_{2}, E_{1}\right) \stackrel{f}{\rightarrow} \operatorname{Hom}_{R}\left(E_{2}, E_{2}\right) \cong R, \\
& \operatorname{Hom}_{R}\left(E_{2}, E_{1}\right) \otimes_{R} \operatorname{Hom}_{R}\left(E_{1}, E_{2}\right) \stackrel{g}{\rightarrow} \operatorname{Hom}_{R}\left(E_{1}, E_{1}\right) \cong R,
\end{aligned}
$$

defined by $f(\sigma \otimes \tau)=\sigma \tau$ and $g(\tau \otimes \sigma)=\tau \sigma$ for every $\sigma \in P$ and every $\tau \in Q$, respectively, are isomorphisms.

Since $E_{1}$ is an injective cogenerator, $f$ is an isomorphism if and only if when $\operatorname{Hom}_{R}\left(-, E_{1}\right)$ is applied, the resultant morphism

$$
\operatorname{Hom}_{R}\left(R, E_{1}\right) \cong E_{1} \longrightarrow \operatorname{Hom}_{R}\left(\operatorname{Hom}_{R}\left(E_{1}, E_{2}\right) \otimes_{R} \operatorname{Hom}_{R}\left(E_{2}, E_{1}\right), E_{1}\right)
$$

is also an isomorphism. But since $E_{1}$ is $E_{1}$-reflexive, by Corollary $1.7, E_{1}$ is also $E_{2}$ reflexive and vice versa, and therefore we get that

$$
\begin{aligned}
\operatorname{Hom}_{R} & \left(\operatorname{Hom}_{R}\left(E_{1}, E_{2}\right) \otimes_{R} \operatorname{Hom}_{R}\left(E_{2}, E_{1}\right), E_{1}\right) \\
& \cong \operatorname{Hom}_{R}\left(\operatorname{Hom}_{R}\left(E_{1}, E_{2}\right), \operatorname{Hom}_{R}\left(\operatorname{Hom}_{R}\left(E_{2}, E_{1}\right), E_{1}\right)\right) \\
& \cong \operatorname{Hom}_{R}\left(\operatorname{Hom}_{R}\left(E_{1}, E_{2}\right), E_{2}\right) \cong E_{1}
\end{aligned}
$$

and it follows that $f$ is an isomorphism. For $g$, the reasoning is analogous.

(ii) Since $g$ is an isomorphism, in particular, $g$ is an epimorphism, and so

$$
1=\sum_{i \in I} \tau_{i} \sigma_{i}
$$

with $\sigma_{i} \in P$ and $\tau_{i} \in Q$ for all $i$. Now let $h_{i}: P \rightarrow R$ defined by $h_{i}(\sigma)=\tau_{i} \sigma$ for each $i$. Then, we have $h: P^{(I)} \rightarrow R$ and by (2.6) it follows that $P$ is a generator. Analogous reasoning is used for $Q$.

(iii) Let $h: P \rightarrow R^{(I)} \cong \operatorname{Hom}_{R}\left(E_{1}, E_{1}\right)^{(I)}$ and let $h^{\prime}: R^{(I)} \rightarrow P$ defined by $h(\sigma)=$ $\left(\sigma \tau_{i}\right)_{i \in I}$ and $h^{\prime}\left(\left(r_{i}\right)_{i \in I}\right)=\sum_{i \in I} r_{i} \sigma_{i}$. It is clear now that $\left(h^{\prime} h\right)(\sigma)=h^{\prime}\left(\left(\sigma \tau_{i}\right)_{i \in I}\right)=$ $\sum_{i \in I}\left(\sigma \tau_{i}\right) \sigma_{i}=\sigma \sum_{i \in I} \sigma_{i} \tau_{i}=\sigma$ using (2.6). It follows that $P \cong R^{(I)}$, and so $P$ is finitely generated projective. Similarly, we prove that $Q$ is finitely generated projective.

(iv) Let $h: P \rightarrow \operatorname{Hom}_{R}\left(Q_{R}, R_{R}\right)$ defined by $h(\sigma)(\tau)=\sigma \tau$ for all $\sigma \in P$ and $\tau \in Q$. If $h(\sigma)=0$ then we get that $\sigma \tau=0$ for all $\tau \in Q$. But $\sigma=\sigma 1=\sigma\left(\sum_{i \in I} \boldsymbol{T}_{i} \sigma_{i}\right)=$ $\sum_{i \in I} \sigma\left(\tau_{i} \sigma_{i}\right)=\sum_{i \in I}\left(\sigma \tau_{i}\right) \sigma_{i}=0$, so $h$ is injective.

Let now $\varphi \in \operatorname{Hom}_{R}(Q, R)$. Then for all $\tau \in Q$,

$$
\begin{aligned}
\varphi(\tau) & =\varphi(1 \tau)=\varphi\left(\left(\sum_{i \in I} \tau_{i} \sigma_{i}\right) \tau\right)=\varphi\left(\sum_{i \in I}\left(\tau_{i} \sigma_{i}\right) \tau\right) \\
& =\varphi\left(\sum_{i \in I} \tau_{i}\left(\sigma_{i} \tau\right)\right)=\sum_{i \in I} \varphi\left(\tau_{i}\right)\left(\sigma_{i} \tau\right) \\
& =\sum_{i \in I}\left(\varphi\left(\tau_{i}\right) \sigma_{i}\right) \tau=h(\sigma)(\tau)
\end{aligned}
$$


where $\sigma=\sum_{i \in I}\left(\varphi\left(\tau_{i}\right) \sigma_{i}\right)$. It follows that $P \cong \operatorname{Hom}_{R}(Q, R)$. Similarly, we can prove that $Q \cong \operatorname{Hom}_{R}\left(P_{R}, R_{R}\right)$.

Now by analogy with the commutative case, we give the following definition.

Definition 2.3. Let $R$ be a ring and let $P$ be an $(R, R)$-bimodule. We will say that $P$ is invertible if it is left and right finitely generated projective and if $P \otimes_{R} \operatorname{Hom}_{R}(P, R) \cong R$ and $\operatorname{Hom}_{R}(P, R) \otimes_{R} P \cong R$.

THEOREM 2.4. Let $R$ be a ring admitting Matlis duality. Then there exists a bijective correspondence between Matlis dualizing modules and invertible modules.

Proof. Let $M$ be a left (right) $R$-module and denote $M^{*}=\operatorname{Hom}_{R}(M, R)$. Note first that, if $F$ is a finitely generated free module, then the natural morphism

$$
F^{*} \otimes_{R} N \stackrel{f}{\rightarrow} \operatorname{Hom}_{R}(F, N)
$$

defined by $f(g \otimes n)=h \in \operatorname{Hom}_{R}(F, N)$ where $h(x)=g(x) n$ is clearly an isomorphism. It follows then that $P^{*} \otimes_{R} N \cong \operatorname{Hom}_{R}(P, N)$ for $P$ finitely generated projective.

We show now that if $P$ is invertible and $E$ is Matlis dualizing, then $P^{*} \otimes E$ is also Matlis dualizing. First, since $P$ is finitely generated projective, so is $P^{*}$. Therefore $P^{*} \otimes_{R} E$ is a direct summand of $R^{n} \otimes_{R} E \cong E^{n}$ for some positive integer $n$ which is injective, and so $P^{*} \otimes_{R} E$ is also injective.

Now if $N$ is any (left or right) $R$-module,

$$
\operatorname{Hom}_{R}\left(N, P^{*} \otimes_{R} E\right) \cong \operatorname{Hom}_{R}\left(N, \operatorname{Hom}_{R}(P, E)\right) \cong \operatorname{Hom}_{R}\left(N \otimes_{R} P, E\right)
$$

which is not zero since $E$ is a cogenerator, this shows that $P^{*} \otimes_{R} E$ is also a cogenerator. Moreover,

$$
\begin{aligned}
\operatorname{Hom}_{R}\left(P^{*} \otimes_{R} E, P^{*} \otimes_{R} E\right) & \cong \operatorname{Hom}_{R}\left(E, \operatorname{Hom}_{R}\left(P^{*}, P^{*} \otimes E\right)\right) \\
& \cong \operatorname{Hom}_{R}\left(E, \operatorname{Hom}_{R}\left(P^{*}, \operatorname{Hom}_{R}(P, E)\right)\right) \\
& \cong \operatorname{Hom}_{R}\left(E, \operatorname{Hom}_{R}\left(P^{*} \otimes P, E\right)\right) \\
& \cong \operatorname{Hom}_{R}\left(E, \operatorname{Hom}_{R}(R, E)\right) \cong \operatorname{Hom}_{R}(E, E) \cong R
\end{aligned}
$$

which shows that $P^{*} \otimes_{R} E$ is a Matlis dualizing module.

On the other hand, if $\bar{E}$ is a Matlis dualizing module, then by Theorem 2.2 we have that $\operatorname{Hom}_{R}(\bar{E}, E)$ is invertible.

If we denote by $\mathscr{D}$ and $\mathscr{I}$, respectively the classes of Matlis dualizing and invertible modules, the correspondence may be then represented by the following diagram:

$$
\mathscr{D} \underset{(-)^{*} \otimes_{R} E}{\stackrel{\operatorname{Hom}_{R}(-, E)}{<}} \mathscr{\longrightarrow} .
$$


If $\bar{E}$ is Matlis dualizing, then

$$
\begin{aligned}
\left((-)^{*} \otimes_{R} E\right) \circ(\operatorname{Hom}(-, E))(\bar{E}) & =\operatorname{Hom}_{R}\left(\operatorname{Hom}_{R}(\bar{E}, E), R\right) \otimes_{R} E \\
& \cong \operatorname{Hom}_{R}\left(\operatorname{Hom}_{R}(\bar{E}, E), E\right) \cong \bar{E}
\end{aligned}
$$

since $\operatorname{Hom}_{R}(\bar{E}, E)$ is finitely generated projective and $\bar{E}$ is $E$-reflexive by Corollary 1.7. Now, if $P$ is invertible,

$$
\left(\operatorname{Hom}_{R}(-, E)\right) \circ\left((-)^{*} \otimes_{R} E\right)(P)=\operatorname{Hom}_{R}\left(P^{*} \otimes_{R} E, E\right) \cong \operatorname{Hom}_{R}\left(\operatorname{Hom}_{R}(P, E), E\right) \cong P
$$

since $P$ is finitely generated projective and also is $E$-reflexive.

REMARK 2.5. Note that all elements in $\mathscr{I}$ are finitely generated projective, and so, by the proof of Theorem $2.4(-)^{*} \otimes_{R} E=\operatorname{Hom}_{R}(-, E)$, so in other words, if $R$ has a Matlis dualizing module $E$, the theorem shows that, in this case, there is a duality between Matlis dualizing modules and invertible modules, which determines the Picard group of $R$.

ACKNOWLEDGMENTS. This paper was written while the second author was visiting University of Lexington, Kentucky, and was supported by Ministerio de Educacion y Cultura grant EX 00 34845300. Second and third authors are partially supported by DGES grant PB98-1005 and Junta de Andalucía FQM 0211.

\section{REFERENCES}

[1] F. W. Anderson and K. R. Fuller, Rings and Categories of Modules, 2nd ed., Graduate Texts in Mathematics, vol. 13, Springer-Verlag, New York, 1992.

[2] J. Dieudonné, Remarks on quasi-Frobenius rings, Illinois J. Math. 2 (1958), 346-354.

[3] E. E. Enochs, Flat covers and flat cotorsion modules, Proc. Amer. Math. Soc. 92 (1984), no. 2, 179-184.

[4] E. E. Enochs, O. M. G. Jenda, and J. Xu, Lifting group representations to maximal CohenMacaulay representations, J. Algebra 188 (1997), no. 1, 58-68.

[5] A. S. McKerrow, On the injective dimension of modules of power series, Quart. J. Math. Oxford Ser. (2) 25 (1974), 359-368.

[6] B. L. Osofsky, A generalization of quasi-Frobenius rings, J. Algebra 4 (1966), 373-387, Erratum, J. Algebra 9 (1968), 120.

[7] S. Park, Inverse polynomials and injective covers, Comm. Algebra 21 (1993), no. 12, 45994613.

[8] W. Xue, Rings with Morita Duality, Lecture Notes in Mathematics, vol. 1523, SpringerVerlag, Berlin, 1992.

EdGAR E. ENOCHS: DePARTMENT OF MATHEMATICS, UNIVERSity OF KeNTUCKY, LeXINGTON, KY 40506, USA

E-mail address: enochs@ms . uky.edu

J. A. López-RAmos: Departamento de Algebra y ANÁlisis MATEmÁtico, Universidad DE ALMERÍA, 04120 ALMERÍA, SPAIN

E-mail address: j1opez@ual.es

B. TORRECILlas: Departamento De Algebra y ANÁlisis MatemÁtico, UniVersidad DE ALMERÍA, 04120 ALMERÍA, SPAIN

E-mail address: btorreci@ual.es 


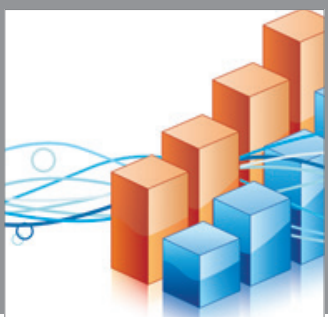

Advances in

Operations Research

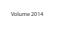

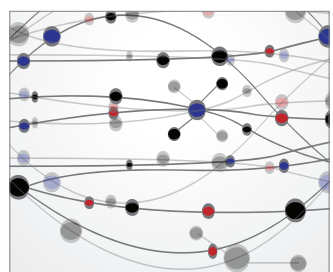

\section{The Scientific} World Journal
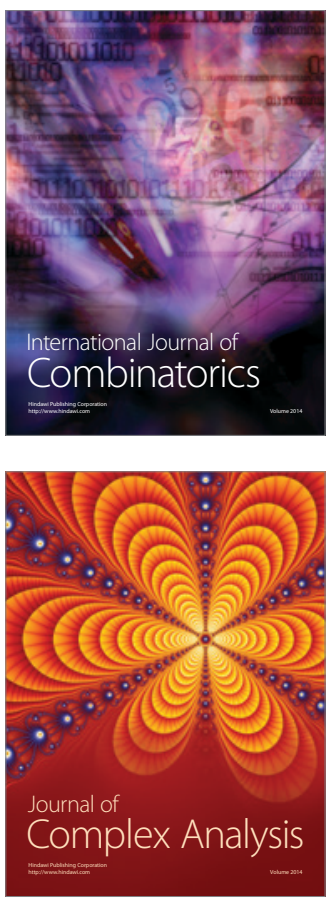

International Journal of

Mathematics and

Mathematical

Sciences
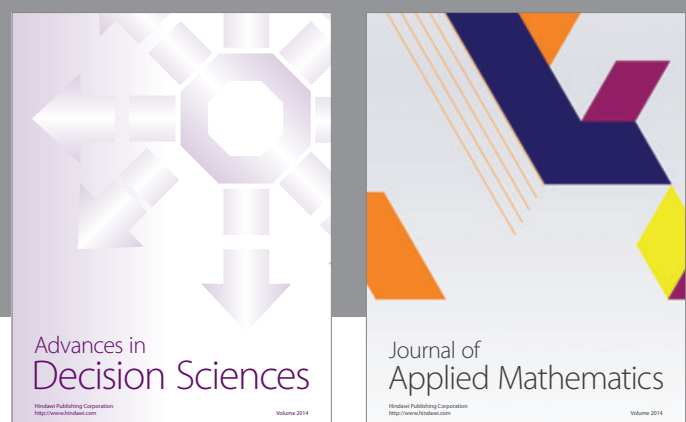

Journal of

Applied Mathematics
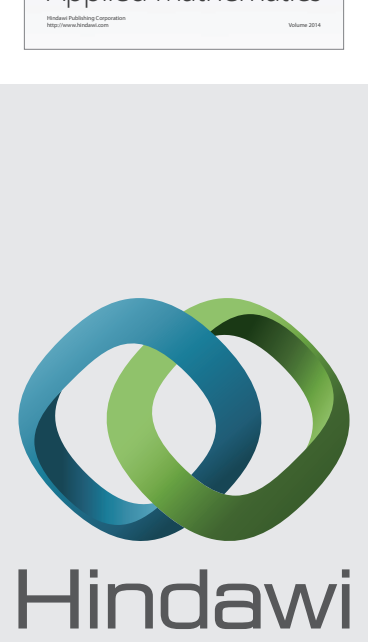

Submit your manuscripts at http://www.hindawi.com
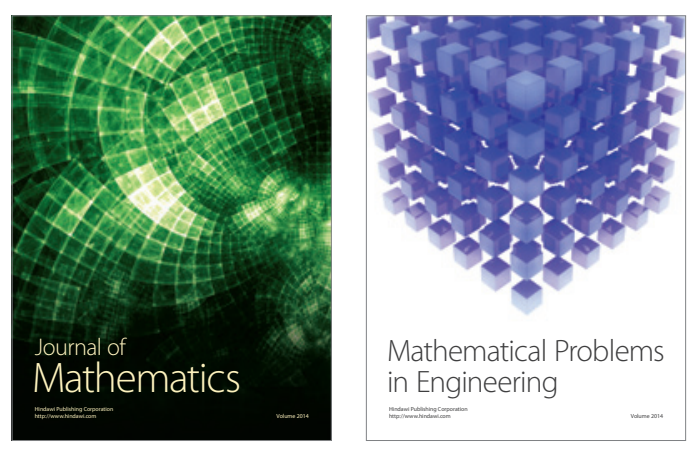

Mathematical Problems in Engineering
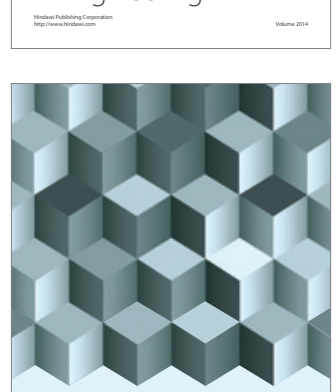

Journal of

Function Spaces
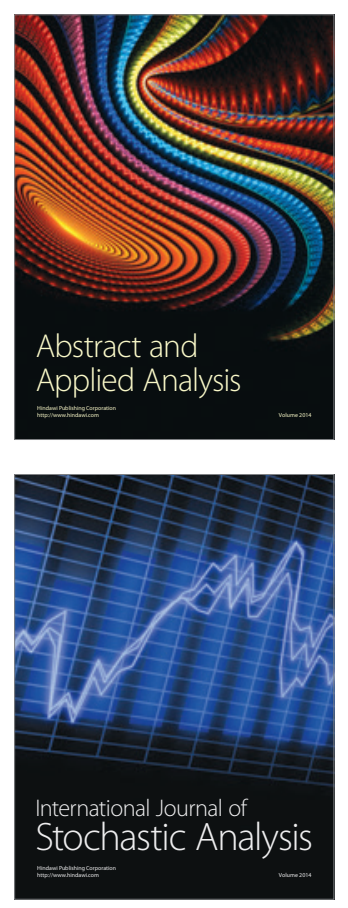

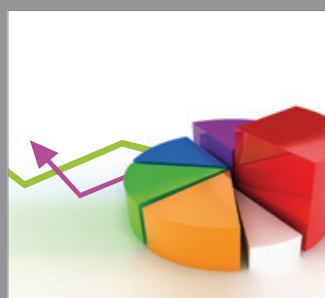

ournal of

Probability and Statistics

Promensencen
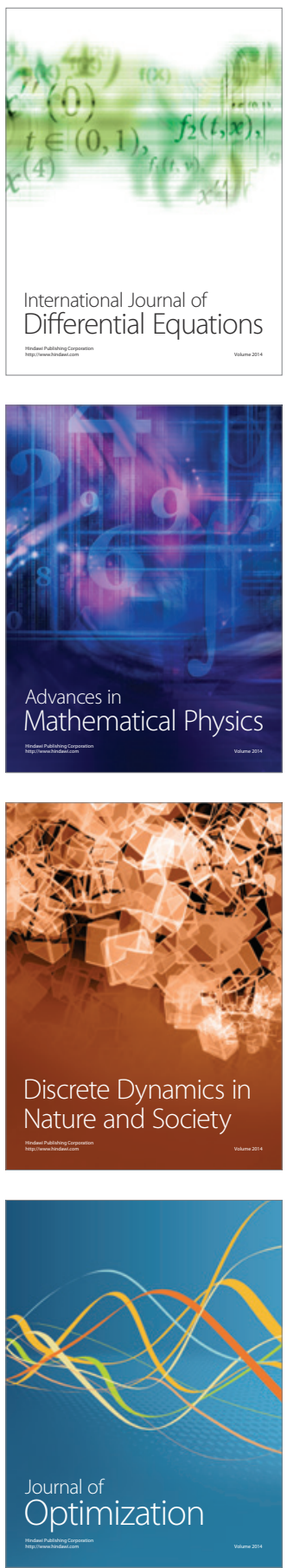